\title{
False-positive HIV DNA PCR testing of infants: Implications in a changing epidemic
}

\author{
Ute Feucht, Brian Forsyth, Mariana Kruger
}

Aim. To examine false-positive HIV DNA polymerase chain reaction (PCR) test results in children, and the potential implications for the paediatric HIV epidemic in sub-Saharan Africa.

Methods. A review was done of records over a 6-year period of children less than 18 months old at an HIV treatment site in South Africa, to evaluate those with an initial 'false'-positive HIV DNA PCR result, but later proven to be HIV-uninfected with HIV DNA PCR and/or quantitative HIV RNA PCR tests. We calculated the influence of changing HIV transmission rates on predictive values (PV) of HIV DNA PCR tests in a hypothetical population of all HIV-exposed infants over a 1-year period. (Positive PV: proportion of individuals with a positive test with disease; negative PV: proportion of individuals with negative test and no disease).

Results. Of 718 children, 40 with an initial positive HIV DNA PCR test were subsequently proven to be HIV-uninfected, resulting in a positive PV of $94.4 \%$. Most (75\%) uninfected children had PMTCT interventions and were asymptomatic or mildly symptomatic (77.5\%). Calculations using a test specificity of $99.4 \%$, as reported previously, show a decrease in positive PV using a single-test strategy from $98.6 \%$ at $30 \%$ HIV transmission rate, to $94.8 \%$ at $10 \%$ transmission, to $62.5 \%$ at $1 \%$ transmission. Reduction in test specificity further decreases positive PV at low transmission rates.

Conclusion. Decreasing mother-to-child HIV transmission rates reduce the positive predictive value of a single HIV DNA PCR test result, necessitating adaptations to diagnostic algorithms to avoid misdiagnosis and inappropriate treatment, especially with early initiation of antiretroviral therapy in asymptomatic infants.

S Afr Med J 2012;102:149-152.
The HIV-pandemic threatens health and life in sub-Saharan Africa, with current prevention methods unable to stem the tide of new adult HIV-infections. ${ }^{1}$ Linked to the adult epiemic is the childhood epidemic, with mother-to-child transmission (MTCT) one of the main modes of transmission. Nearly a third of all children are born to HIV-infected women in high-prevalence areas, such as South Africa, which has major implications for improved child survival. ${ }^{2}$ Contrasting with the adult epidemic, the childhood epidemic can be effectively reduced through prevention-of-mother-to-child (PMTCT) interventions, decreasing the risk of transmission from $35 \%$ to below $5 \% .^{3}$ Low- and middle-income countries have faced operational challenges in offering PMTCT services to all at-risk mother-infant pairs but, in South Africa, a new PMTCT era is dawning, with intensified PMTCT guidelines released in 2010.,5

As HIV disease progression in children is rapid, its impact on childhood morbidity and mortality will only be decreased if HIVinfected children are diagnosed early and antiretroviral therapy (ART) initiated in infancy. ${ }^{6,7}$ South Africa released national HIV treatment guidelines in 2004, opening ART access to public sector patients. ${ }^{8}$ Diagnosis of HIV infection in adults and children $>18$ months old is by HIV ELISA or rapid tests, verifying a positive screening test with a confirmatory test. In children $<18$ months old, persistence of maternal HIV antibodies means diagnosis relies on

Department of Paediatrics, Kalafong Hospital, University of Pretoria Ute Feucht, MB ChB, FC (Paed) SA, MMed (Paed), Dip HIV Man (SA), CAHM

Department of Pediatrics, Yale University, New Haven, CT, USA Brian Forsyth, MB ChB, FRCP (C)

Department of Paediatrics and Child Health, University of Stellenbosch Mariana Kruger, MB ChB, MMed (Paed), MPhil, PhD virological assays, with HIV DNA PCR (polymerase chain reaction) testing most commonly used. ${ }^{8}$ Sensitivity and specificity of a single HIV DNA PCR test is excellent at $98.8 \%$ and $99.4 \%$ within a research environment in Johannesburg. ${ }^{9}$ Diagnostic services must be available for children to access care, and HIV DNA PCR testing is much more costly and less widely available than HIV ELISA/rapid testing. ${ }^{10}$ With PMTCT programme expansion, infant HIV testing has shifted to primary health care facilities using the dry blood spot (DBS) technique, aiming at testing all HIV-exposed infants at 6 weeks of age. ${ }^{11,12}$ The SA PMTCT guidelines (2010) recommend that a positive HIV DNA PCR test is confirmed by HIV RNA viral load testing. ${ }^{4}$

To predict the probability of actual disease, clinicians rely on the sensitivity, specificity, positive predictive value (PPV) and negative predictive value (NPV) of diagnostic tests. The sensitivity of any test is the proportion of people with a positive test among those who have the disease, while specificity is the proportion of people with a negative test among those without the disease. The PPV is the proportion of people with disease among all those with a positive test, and the NPV is the proportion of people without the disease among those with a negative test result. Both the PPV and NPV depend on the prevalence of the disease in the tested population. The predictive value is the key characteristic, as it gives information about how likely it is that the test result correlates with actual disease. ${ }^{13}$

We examined our experience of referrals of children incorrectly diagnosed as HIV-positive to illustrate the potential implications in a changing epidemic within the context of PMTCT programme expansion, decreasing HIV transmission rates and an emphasis on initiating ART in early infancy before children are symptomatic.

\section{Patients and methods}

The Kalafong regional hospital serves an urban population in Gauteng Province, and delivers ART care to public sector patients. In this retrospective analysis, we included all records of children $<18$ months old referred to the hospital's HIV clinic between April 2004 and March 2010, which is the period from the start of the national ART programme to the implementation of the updated 2010 guidelines. The only records excluded were of those initiated on ART 
before referral to Kalafong. The Ethics Review Committee, Faculty of Health Sciences, University of Pretoria, approved the study protocol. The patient records of all the discharged children after an initial false-positive HIV DNA PCR test were reviewed by a paediatrician with extensive experience in HIV care. Efforts were made to trace the children who were discharged as HIV-uninfected.

The samples for the initial HIV DNA PCR tests were taken either at the Kalafong PMTCT follow-up clinic or at regional primary health care clinics. One virology laboratory of the National Health Laboratory Service (NHLS), linked to a tertiary training hospital, processed all HIV DNA PCR tests, using the AMPLICOR HIV-1 DNA Test, version 1.5 (Roche Molecular Systems, Inc.) from 2004 until 2009, and the Cobas AmpliPrep/Cobas TaqMan HIV-1 Qual test (Roche Molecular Systems, Inc.) thereafter. Standard practice in the clinic was to do a confirmatory HIV DNA PCR test and/ or quantitative HIV RNA PCR ('viral load') on all newly referred children $<18$ months. Additional HIV-testing in children with an initial positive HIV DNA PCR result ('false positive HIV DNA PCR test') was prompted by either a negative confirmatory HIV DNA PCR test or the HIV viral load (quantitative HIV RNA PCR) result being undetectable. Further testing depended on the child's age and included additional HIV DNA PCR and viral load tests in children $<18$ months, and HIV ELISA testing in children $\geq 18$ months. The decision to discharge the child as HIV-negative was made by the treating clinician. Children who at discharge, owing to their young age, had not yet had an HIV ELISA test done, were referred to primary health care for HIV rapid or ELISA testing at 18 months.

Using estimates of the number of HIV-exposed infants born in South Africa during a one-year period, calculations examined the influence of changing HIV transmission rates on the PPV and NPV of a single HIV DNA PCR test. The literature provided data regarding the number of HIV-exposed infants born in South Africa within a one-year period and the sensitivity and specificity of the HIV DNA PCR test commonly used within the local context.

\section{Results}

In 40 of the 718 children aged $<18$ months at the first Kalafong clinic visit, the initial positive HIV DNA PCR test was not confirmed by a second HIV DNA PCR $(N=12)$ or HIV RNA PCR test $(N=20)$ or both tests (DNA and RNA PCR) $(N=8)$. The median age of all patients at first clinic visit was 7 months (range 26 days to 17 months), while the median age of those with initial positive HIV DNA PCR results, not confirmed subsequently ('false positive'), was 4 months (range 1 month - 35 months; one child aged 35 months was referred late owing to lost-to-follow-up after initial positive HIV DNA PCR result in infancy). The ratio of male and female infants referred to the clinic was 1.08 to 1 but, for those with false-positive results, males predominated (ratio 2.08:1) $(p=0.04)$.

At HIV re-testing, 15 (37.5\%) children subsequently had 2 negative virological tests, and $10(25 \%)$ had $\geq 3$ negative virological assays, which included combinations of negative HIV DNA PCR and undetectable viral load results. Another 15 (37.5\%) were discharged after at least 1 negative HIV ELISA test in addition to $\geq 1$ negative HIV virological assays (negative HIV DNA PCR test or undetectable HIV viral load).

Review of the medical records indicated no discharge to have been inappropriate, and none of the discharged children was later referred back to the clinic as HIV-infected. Telephonic follow-up was conducted in August 2010, and none of the 22 (55\%) children who were traceable had subsequently been diagnosed as HIV-infected.

As part of the confirmatory testing, $49 \mathrm{HIV}$ viral loads were done on venous blood on 33 of the HIV-uninfected children, of which 40
(82\%) were undetectable, while 8 in total had 9 detectable viral load results: 6 (12\%) were between $\log _{10} 2$ and 3 , and $3(6 \%)$ were between $\log _{10} 3$ and 4 , with none above $\log _{10} 4$ (10 000 copies). The median age at initial positive HIV DNA PCR test for this subgroup was 1.5 months (range 11 days - 9 months), while the median age of the detectable HIV viral load was 10 months (range 1 - 46 months). Five of the 8 children with detectable viral loads, including the one child with low detectable viral loads on 2 occasions, were subsequently HIV ELISA non-reactive, while the other 3 children, all discharged before age 18 months, had 2 subsequent negative virological assays to exclude HIV infection.

Of the uninfected children, 30 (75\%) had recorded PMTCT interventions, while $5(12.5 \%)$ had no PMTCT interventions, and another $5(12.5 \%)$ had no information recorded. Twenty (67\%) mother-infant pairs had received single-dose nevirapine (NVP), 7 (23\%) had mothers on triple ART with subsequent infant NVP, 2 (7\%) had combined zidovudine (AZT) and NVP interventions, and 1 (3\%) had other PMTCT interventions. Twenty-eight (70\%) mothers never breastfed their infants, 5 (12.5\%) had a breastfeeding history, while in 7 (17.5\%) this information was not recorded or not known. According to WHO HIV clinical staging done at the HIV clinic, 31 (77.5\%) were asymptomatic or had a minor illness (stage 1 and 2), 7 ( $17.5 \%)$ were classified as symptomatic (with stage 3 or 4 disease), and $2(5 \%)$ had not been staged.

In summary: with 40 (out of 718) unconfirmed positive HIV DNA PCR tests and 678 (out of 718) confirmed positive tests, the PPV of the HIV DNA PCR test in the clinical setting at Kalafong Hospital in infants $<18$ months was calculated at $94.4 \%$. Reasons for these false-positive results can arise from specimen problems or inherent laboratory assay performance, which was not possible to investigate retrospectively, and we therefore continued to examine the relevance of these local results within the wider South African context with a changing HIV epidemic in children.

\section{Examination of the results in the wider South African context}

South Africa has a rapidly changing paediatric HIV epidemic, with decreasing transmission rates as a result of intensified PMTCT interventions. The national HIV prevalence among SA pregnant women in 2008 was $29.3 \%$ (95\% confidence interval (CI) 28.5 $30.1 \%),{ }^{2}$ and with a total of 1069300 live births ${ }^{14}$ this means that an estimated $313305 \mathrm{HIV}$-exposed children were born during that year.

The sensitivity and specificity of a single HIV DNA PCR test in a research setting in South Africa is respectively $98.8 \%$ and 99.4\%. ${ }^{9}$ We used this sensitivity and specificity to estimate the number of infants testing HIV DNA PCR positive and negative with varying HIV transmission rates. Table I shows that the number of positive results that are false-positives increases as the HIV transmission rate decreases. The PPV remains relatively high when the transmission rate is $>10 \%$, but drops substantially once the transmission rate is $<10 \%$. Conversely the number of false-negative results decreases as the transmission rate decreases, but the NPV remains very high at all transmission rates, approaching $100 \%$ with very low transmission rates.

Fig. 1 demonstrates the effect on PPV if the specificity of the test were to be less accurate when used in clinical setting than the $99.4 \%$ found in the research environment. When the transmission rate is high, the PPV remains high at all levels of specificity; but once the transmission rate decreases, PPV decreases markedly with even a minor decrease in the specificity of the test by one or two percentage points. A similar decrease in the test sensitivity only marginally affects the NPV. 
Table I. Hypothetical HIV DNA PCR results with predictive values in South African HIV-exposed infants in relation to different HIV transmission rates $\left(\%\right.$ MTCT) ${ }^{\star}$

\begin{tabular}{|c|c|c|c|c|c|c|c|c|}
\hline & \multicolumn{8}{|c|}{ HIV transmission rate (\% MTCT) } \\
\hline & $30 \%$ & $25 \%$ & $20 \%$ & $15 \%$ & $10 \%$ & $5 \%$ & $3 \%$ & $1 \%$ \\
\hline \multicolumn{9}{|c|}{ Estimated absolute numbers of HIV-exposed children tested using a single HIV DNA PCR } \\
\hline \multicolumn{9}{|l|}{ Number of true-positive HIV DNA } \\
\hline PCR tests & 92863 & 77386 & 61909 & 46432 & 30954 & 15477 & 9286 & 3095 \\
\hline \multicolumn{9}{|l|}{ Number of false-positive HIV DNA } \\
\hline PCR tests & 1316 & 1410 & 1504 & 1598 & 1692 & 1786 & 1824 & 1861 \\
\hline \multicolumn{9}{|l|}{ Number of true-negative HIV DNA } \\
\hline PCR tests & 217998 & 233569 & 249140 & 264711 & 280283 & 295854 & 302082 & 308311 \\
\hline \multicolumn{9}{|l|}{ Number of false-negative HIV DNA } \\
\hline PCR tests & 1128 & 940 & 752 & 564 & 376 & 188 & 113 & 38 \\
\hline \multicolumn{9}{|c|}{ Predictive values of a single HIV DNA PCR test result } \\
\hline$\%$ false-positive HIV DNA PCR tests & $1.4 \%$ & $1.8 \%$ & $2.4 \%$ & $3.3 \%$ & $5.2 \%$ & $10.3 \%$ & $16.4 \%$ & $37.6 \%$ \\
\hline Positive predictive value (PPV) & $98.6 \%$ & $98.2 \%$ & $97.6 \%$ & $96.7 \%$ & $94.8 \%$ & $89.7 \%$ & $83.6 \%$ & $62.4 \%$ \\
\hline \% false-negative HIV DNA PCR tests & $0.5 \%$ & $0.4 \%$ & $0.3 \%$ & $0.2 \%$ & $0.1 \%$ & $0.1 \%$ & $0 \%$ & $0 \%$ \\
\hline Negative predictive value (NPV) & $99.5 \%$ & $99.6 \%$ & $99.7 \%$ & $99.8 \%$ & $99.9 \%$ & $99.9 \%$ & $100 \%$ & $100 \%$ \\
\hline
\end{tabular}

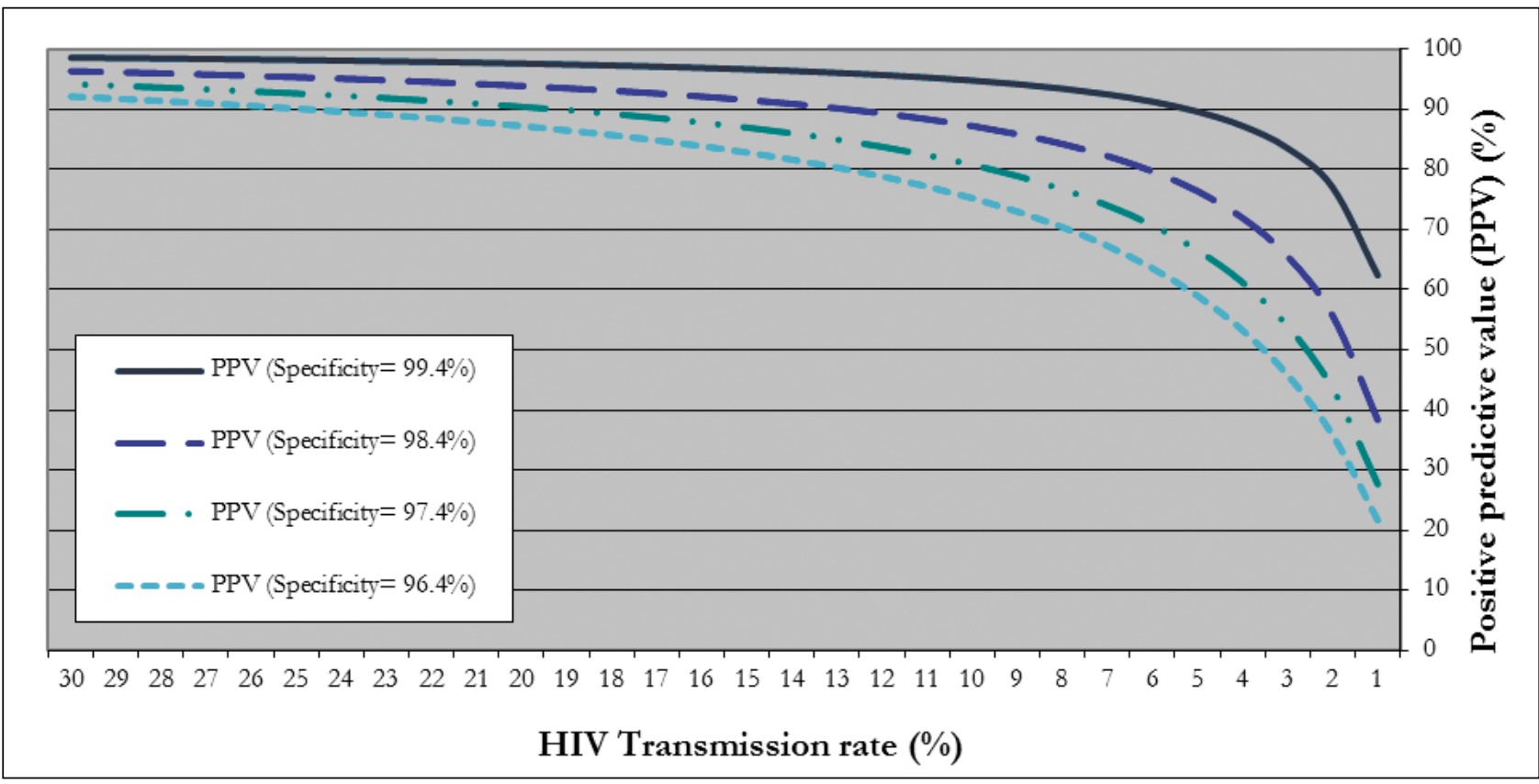

Fig. 1. Positive predictive value (PPV) of the HIV DNA PCR test at varying test specificities and declining HIV transmission rates.

Applying the PPV of $94.4 \%$ found in the Kalafong cohort, Fig. 1 shows that this would correspond to a test specificity of $99.4 \%$ and a transmission rate of between $8 \%$ and $9 \%$, which correlates with the clinical experience in the PMTCT follow-up services at the hospital (data not shown). This conclusion suggests that the test specificity as performed in the clinic is probably holding up to the standards set in the research environment.

\section{Discussion}

ART has transformed paediatric HIV disease into a treatable condition. ${ }^{1}$ Early disease detection and access to ART are priorities to reduce childhood morbidity and mortality in sub-Saharan Africa, especially in children $<1$ year old, with survival benefits even when asymptomatic.? But placing children on ART commits them to lifelong treatment, emphasising the need for an accurate diagnosis before ART initiation.

The HIV DNA PCR test has an excellent sensitivity and specificity within a research setting in South Africa, where much attention is given to having and training adequate staff. ${ }^{9}$ Stretched human resources may compromise test sensitivity and specificity in everyday clinical use, with greater risk of specimen handling errors at clinic and laboratory level. There is also the risk of laboratory errors through specimen contamination and inadequate quality control, as illustrated by our patients with a false-positive viral load and negative confirmatory diagnostic tests. ${ }^{9,11,15}$ 
Our intention was to study how well the HIV DNA PCR test performs in an everyday clinical setting within a large-scale HIV programme. The PPV of the HIV DNA PCR test for children referred to the Kalafong HIV services was $94.4 \%$, which means that 1 in every 18 children $<18$ months old referred as HIV-infected on the basis of 1 positive HIV DNA PCR test result was subsequently determined to be HIV-uninfected, either because of sample misallocation or inherent viral assay performance. The statistically significant gender distribution of more boys than girls having a false-positive HIV DNA PCR test is unexplained.

Although the audit spans a 6-year period in which the PMTCT interventions were not static but expanding, $75 \%$ of children in the HIV-uninfected group had access to PMTCT interventions, thereby greatly reducing their actual risk of being HIV-infected. Most (77.5\%) children with false-positive HIV DNA PCR tests were asymptomatic or only had minor health problems, and only $12.5 \%$ were breastfed, with a risk of late postnatal HIV transmission.

This study's main limitation is that it is a clinic-based retrospective audit. Clinicians might not have identified all children misdiagnosed as being HIV-infected. Reviewing the medical records indicated that none of the discharges as HIV-uninfected was inappropriate. We do not have access to all results of follow-up testing, but all of those known to have had follow-up testing were confirmed uninfected, and none was subsequently referred back to the Kalafong ART site with HIV-disease.

The study spans the time from the first availability of ART in 2004 for children accessing healthcare in the SA public sector up to implementation of the updated guidelines in 2010. One major change to the guidelines is the immediate start of ART for all children diagnosed with HIV before age one year, even if asymptomatic. Their diagnosis of HIV infection then relies solely on positive laboratory testing. ${ }^{16}$

The expanding PMTCT programme has the potential to rapidly shift the epidemiology of HIV disease in children, uncoupling it from the adult epidemic. There is a striking non-linear relationship between decreasing disease prevalence and PPV of the HIV DNA PCR tests, as shown from the calculations of HIV DNA PCR testing in South Africa that show the rapid decrease in PPV of the test when disease prevalence drops below $10 \%$. This means that countries with improving PMTCT programmes must revisit testing algorithms, as the shifting epidemic itself causes major changes in the PPV of the virological assay. Any quality assurance compromise at clinic or laboratory level potentially decreases test specificity, further rapidly decreasing the PPV of a single positive test. Laboratory accreditation and continued quality control are therefore critical. The NPV of the test is excellent, meaning that a child without continued exposure can safely be labelled as HIV-uninfected with one negative test, even within a possible context of compromised laboratory services with decreasing test sensitivity.

HIV testing strategies must differentiate whether testing is done in children who have clinical signs of HIV disease or in asymptomatic children from the PMTCT programme, as testing algorithms must take into account the disease prevalence in the tested population. ${ }^{10,15}$ The laboratory capacity to do HIV testing on all HIV-exposed infants has expanded greatly in countries such as South Africa, but is a potential limiting factor. ${ }^{10}$ Testing algorithms must be cost-effective, with incremental costs added with each additional step whereas, conversely, insufficient testing may compromise individual patient safety. ${ }^{17}$ The WHO recommends a 3-test strategy for asymptomatic people, when the population prevalence is less than $5 \%$, as in asymptomatic infants tested in well-functioning PMTCT programmes. ${ }^{15}$
The updated SA Paediatric HIV guidelines (2010) recommend HIV testing in infants using 1 HIV DNA PCR test, with an HIV RNA viral load above 10000 copies/ml $\left(>\log _{10} 4\right)$ used as a confirmatory test. ${ }^{16}$ In our study, $82 \%$ of HIV viral loads done on children with false-positive HIV DNA PCR results were undetectable, while the false-positive detectable viral loads were all below the threshold of $\log _{10} 4$. Training of healthcare workers is paramount to avoid low-level positive HIV viral load results being interpreted as proof of the HIVpositive status of an uninfected child. ${ }^{11}$

For countries similar to South Africa, with expanding PMTCT interventions aimed at reducing the transmission rate to $<5 \%$ and the laboratory capacity to do HIV DNA PCR tests, it is strongly advised to move to a 3-test strategy and add a confirmatory HIV DNA PCR test to the current testing strategy for asymptomatic infants who have 1 positive HIV DNA PCR test. The additional cost of testing will decrease as HIV prevalence in children declines and there are fewer positive results owing to PMTCT interventions. Also, costs will be recovered by avoiding ART initiation in HIV-uninfected children. Clinicians must guard against a false sense of security when a test has an excellent specificity as, in a setting of low disease prevalence, the PPV of the test is decreased. This is especially true in a changing epidemic, when the risk of infection status is not constant over time, as is the current situation with the paediatric HIV epidemic in sub-Saharan Africa.

Acknowledgements. I thank Ms L Hahne for the development of the electronic database for the clinic; Drs W Thomas and M Poane, and Ms D Sekwakwa, for their assistance with data collection; Professor F Hahne for help with calculations; and Kalafong Paediatric HIV clinic staff for their dedicated service to patients and their assistance with data collection.

\section{References}

1. World Health Organization. Priority interventions: HIV/AIDS Prevention, Treatment and Care in the Health Sector. Geneva: WHO, 2009. http://www.who.int/hiv/pub/priority_interventions_web.pdf (accessed 13 April 2011).

2. Department of Health. 2008 National Antenatal Sentinel HIV \& Syphilis Prevalence Survey, South Africa. http://www.doh.gov.za/docs/reports/index.html (accessed 13 April 2011).

3. World Health Organization. Antiretroviral drugs for treating pregnant women and preventing HIV . World Health Organization. Antiretroviral drugs for treating pregnant women and preventing HIV
infections in infants. Recommendations for a public health approach. Geneva: WHO, 2010. http:// infections in infants. Recommendations for a public health approach. Geneva: WHO, 2010.
www.searo.who.int/LinkFiles/HIV-AIDS_PMTCT_Guidelines.pdf ( accessed 13 April 2011).

4. National Department of Health: Clinical Guidelines: PMTCT (Prevention of Mother-to-Child 4. National Department of Health: Clinical Guidelines: PMTCT (Prevention of Mother-to-Child
Transmission), 2010. www.doh.gov.za/docs/factsheets/guidelines/pmtct.pdf (accessed 13 April 2011). 5. Horwood C. Prevention of mother-to-child HIV transmission in South Africa: the dawning of a new era. HIV Ther 2010;4(2):127-130

6. Bourne DE, Thompson M, Brody LL, et al. Emergence of a peak in early infant mortality due to HIV/ AIDS in South Africa. AIDS 2009;23(1):3-11.

7. Violari A, Cotton MF, Gibb DM, et al for the CHER Study Team. Early antiretroviral therapy and mortality among HIV-infected infants. N Engl J Med 2008;359:2233-2244.

8. National Department of Health: National Antiretroviral Treatment Guidelines. 1st ed, 2004. http:// www.doh.gov.za/docs/hivaids-progressrep.html (accessed 13 April 2011)

9. Sherman GG, Cooper PA, Coovadia AH, et al. Polymerase chain reaction for diagnosis of human immunodeficiency virus infection in infancy in low resource settings. Pediatr Infect Dis J 2005;24:993-997.

10. Creek TL Sherman GG, Nkengasong I et al Infant human immunodeficiency virus diagnosis in resource-limited settings: issues, technologies, and country experiences. Am I Obstet Gynecol 2007;197:S64-S71. (1)

Ginsburg AS, Miller A, Wilfert CM. Diagnosis of pediatric human immunodeficiency virus infection in resource-constrained settings. Pediatr Infect Dis J 2006;25(11):1057-1064

2. Sherman GG, Stevens GPD, Jones SA, Horsfield PMT, Stevens WS. Dried blood spots improve access to HIV diagnosis and care for infants in low resource settings. J Acquir Immune Defic Syndr 2005;38(5):615-617.

13. U.S. Department of Health and Human Services, Centers for Disease Prevention and Control (CDC), World Health Organization, African Regional Office (WHO/AFRO). Guidelines for appropriate evaluations of HIV testing technologies in Africa. http://www.who.int/entity/diagnostics_laboratory/ publications/EN_HIVEval_Guide.pdf (accessed 13 April 2011).

14. Statistics South Africa. Mid-year Population Estimates 2009. www.statssa.gov.za (accessed 13 April 2011).

15. WHO Recommendations on the Diagnosis of HIV Infection in Infants and Children. Geneva: World Health Organization, 2010. http://whqlibdoc.who.int/publications/2010/9789241599085_eng.pdf (accessed 13 April 2011.)

16. National Department of Health. Guidelines for the Management of HIV in Children 2nd ed, 2010. www.doh.gov.za/docs/factsheets/index.html (accessed 13 April 2011).

7. Mrus JM, Yi MS, Eckman MH, Tsevat J. The impact of expected HIV transmission rates on the effectiveness and cost of ruling out HIV infection in infants. Med Decis Making 2002;22:S38-S44. 